\title{
Risk factors for breakthrough urinary tract infection in children with vesicoureteral reflux receiving continuous antibiotic prophylaxis
}

\author{
Dequan Su ${ }^{1, \#}$, Qian Shen ${ }^{1 \#}$, Yihui Zhai ${ }^{1}$, Jing Chen ${ }^{1}$, Jia Rao ${ }^{1}$, Qianfan Miao ${ }^{1}$, Xiaoshan Tang ${ }^{1}$, \\ Zhiqing Zhang ${ }^{1}$, Jiaojiao Liu ${ }^{1}$, Jialu Liu ${ }^{1}$, Hong $\mathrm{Xu}^{1}$, Xiaoyan Fang ${ }^{1}$ \\ ${ }^{1}$ Department of Nephrology, Children's Hospital of Fudan University, Shanghai, China; ${ }^{2}$ Department of Nephrology, Children's Hospital of Fudan \\ University Xiamen Branch, Xiamen, China \\ Contributions: (I) Conception and design: Q Shen, X Fang, H Xu; (II) Administrative support: Q Shen, H Xu; (III) Provision of study materials or \\ patients: Y Zhai, J Chen, J Rao, Q Miao; (IV) Collection and assembly of data: D Su, X Tang, Z Zhang, Jiaojiao Liu, Jialu Liu; (V) Data analysis and \\ interpretation: D Su, X Fang, Q Shen; (VI) Manuscript writing: All authors; (VII) Final approval of manuscript: All authors. \\ \#These authors contributed equally to this work. \\ Correspondence to: Hong Xu; Xiaoyan Fang. Department of Nephrology, Children's Hospital of Fudan University, Shanghai, China. \\ Email: hxu@shmu.edu.cn; ffxy@vip.sina.com.
}

\begin{abstract}
Background: To investigate the risk factors for breakthrough urinary tract infection (BT-UTI) in children with vesicoureteral reflux (VUR) receiving continuous antibiotic prophylaxis (CAP).

Methods: This was a single-centre cohort study (January 2016 to December 2019). The clinical data of 256 children with grade I-V VUR receiving CAP were analysed. In this study, exposure variables were sex, younger age at the initial diagnosis of UTI $\leq 12$ months, high-grade VUR, bilateral VUR, aetiology, presence of renal scarring at the initial diagnosis, presence of renal function impairment at the initial diagnosis, ultrasound abnormalities, antibiotic used and bladder and bowel dysfunction (BBD). Outcome was BT-UTI.

Results: BT-UTI occurred in 81 out of 256 children with grade I-V VUR who received CAP, an incidence of $31.64 \%$. Univariate analysis showed that younger age at the initial diagnosis of UTI ( $\leq 12$ months), bilateral VUR, renal scarring on the dimercaptosuccinic acid (DMSA) scan at the initial diagnosis of UTI and BBD were correlated with the occurrence of BT-UTI. Multivariate analysis showed that younger age at the initial diagnosis of UTI ( $\leq 12$ months) [hazard ratio (HR): 4.629; 95\% confidence interval (CI): $1.302-$ 16.462], bilateral VUR (HR: 2.078; 95\% CI: 1.084-4.022) and BBD (HR: 3.194; 95\% CI: 1.243-8.206) were independent risk factors for the occurrence of BT-UTI.
\end{abstract}

Conclusions: For VUR children receiving CAP, younger age at the initial diagnosis of UTI ( $\leq 12$ months), bilateral VUR, and BBD were independent risk factors for the occurrence of BT-UTI.

Keywords: Vesicoureteral reflux (VUR); continuous antibiotic prophylaxis (CAP); breakthrough urinary tract infection (BT-UTI); children; bladder and bowel dysfunction (BBD)

Submitted Aug 22, 2021. Accepted for publication Dec 22, 2021.

doi: $10.21037 /$ tp-21-398

View this article at: https://dx.doi.org/10.21037/tp-21-398

\section{Introduction}

Vesicoureteral reflux (VUR) is a common congenital anomaly of the kidney and urinary tract in children. Children with VUR have a high risk of urinary tract infection (UTI), and $5-10 \%$ of children can have continuous renal injury and renal scarring, leading to the formation of reflux nephropathy, which eventually results in hypertension and end-stage renal disease (1-4). The current treatment strategies were designed to reduce new renal injury and 


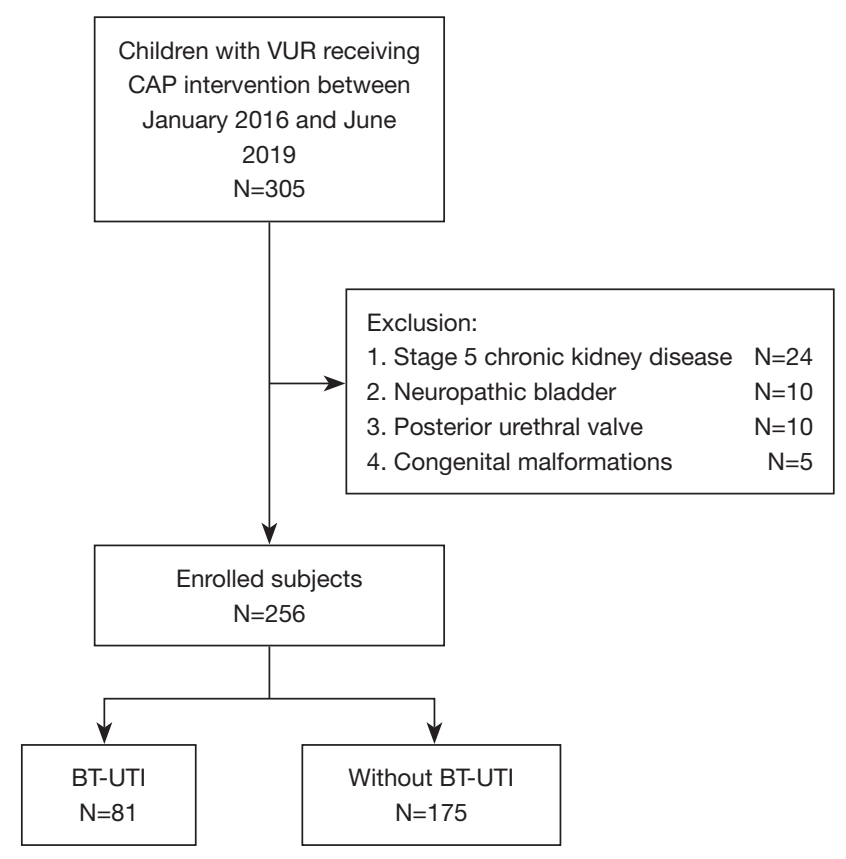

Figure 1 Selection and group information of the study subjects. VUR, vesicoureteral reflux; CAP, continuous antibiotic prophylaxis; BT-UTI, breakthrough urinary tract infection.

prevent new UTIs $(5,6)$. Current, there are two kinds of interventions for VUR patients, continuous antibiotic prophylaxis (CAP) and anti-reflux surgery. Anti-reflux surgery is based on the early published studies suggesting that VUR is unlikely to heal spontaneously. In recent years, it has been reported that patients with intermediate and high-grade VUR have higher spontaneous remission rates than expected (7). Our preliminary study was the first to report the long-term spontaneous remission rate of VUR in China and found that the spontaneous remission rate reached $44.4-56.2 \%$ within 2 years after diagnosis, even in children with severe VUR $(2,8)$. The rate of spontaneous remission of VUR, especially in the first year after the diagnosis, was reported to be higher abroad (9). The guidelines developed by the American Urological Association and European Association of Urology still recommend CAP as the preferred treatment for most children with VUR. CAP can minimize bacterial growth and avoid breakthrough urinary tract infection (BT-UTI) as much as possible, thereby reducing the renal scarring (10-12). A meta-analysis on the role of CAP in children with VUR showed that CAP was effective in preventing the recurrence of BT-UTI (13). Despite the use of CAP, some VUR patients still experience BT-UTIs, thus requiring anti- reflux surgery. The development of clinical strategies on the treatment course and indications of CAP intervention in VUR patients, the effects of CAP on reducing the occurrence of BT-UTI and renal scarring to protect renal function, and the appropriate timing of VUR surgery still requires more clinical evidence-based information.

We present the following article in accordance with the STROBE reporting checklist (available at https:// tp.amegroups.com/article/view/10.21037/tp-21-398/rc).

\section{Methods}

This was a retrospective cohort study. Children seeking treatment due to febrile UTI at the outpatient clinic or inpatient ward of the Department of Nephrology, Children's Hospital of Fudan University, between January 2016 and June 2019 were enrolled in the study. These children were diagnosed with VUR by micturating cystourethrography (MCU) and received CAP intervention. The exclusion criteria were as follows: secondary VUR (neuropathic bladder, posterior urethral valve, congenital tethered cord syndrome), stage 5 chronic kidney disease, genital malformations (urethro-rectal fistula, recto-vesical fistula), and congenital immunodeficiency. A total of 305 children were enrolled in the study. According to the exclusion criteria, 256 children were included in the BT-UTI risk factor analysis (Figure 1). All subjects were diagnosed with VUR by MCU after their febrile UTI was relieved. The diagnosis and grading were based on the VUR classification (grade I-V) developed by the International Reflux Study Committee (14). The classification of bilateral VUR was determined according to the side with a higher reflux grade. After the diagnosis of VUR, all patients received CAP. CAP was given in a single oral dose per night (one-quarter to one-third of a daily dose). Antibiotics (one of nitrofurantoin enteric-coated tablets, sulfamethoxazole tablets, amoxicillin and cephalosporin) were selected according to the bacterial susceptibility and were orally administered. The treatment was individualized for each child. Kidney dimercaptosuccinic acid (DMSA) scan interpretation: ${ }^{99 \mathrm{~m}} \mathrm{Tc}-$ DMSA renal static imaging was performed. After receiving an intravenous injection of 22.2-185.0 MBq imaging agent $(1.85 \mathrm{MBq} / \mathrm{kg}$ body weight) for $3-4 \mathrm{~h}$, children were placed in the supine position, and static imaging in the anteroposterior view was performed using a low-energy high-resolution parallel-hole collimator with a field of view that included both kidneys and the bladder, energy peak $140 \mathrm{keV}$, window width $20 \%$, matrix $512 \times 512$, magnification 
2.19 , and radioactivity count $1.8 \mathrm{mSv}$. The ${ }^{99 \mathrm{~m}} \mathrm{Tc}-\mathrm{DMSA}$ renal imaging protocol and result assessment followed the clinical guideline of the Society of Nuclear Medicine and Molecular Imaging (15). Normal imaging was considered to be a normal location, morphology, and contour of both kidneys and a uniform distribution of radioactivity in the renal parenchyma. The percentage of renal function on each side (differential renal function) was calculated by delineating the kidney regions of interest and background through computer. A kidney with a differential function $<45 \%$ was considered renal impairment. In addition, then the eGFR $<90 \mathrm{~mL} /\left(\min \cdot 1.73 \mathrm{~m}^{2}\right)$ was also defined renal impairment, which calculated by the Schwartz formula $(2,16)$. One or more focal radioactivity reductions or defects in the renal cortex, with contraction or volume reduction of the affected cortex, or wedge-shaped defects denoted renal scarring. Bladder and bowel dysfunction (BBD): a total of $54 \mathrm{VUR}$ children who received potty training during the followup of this cohort were evaluated with the Dysfunctional Voiding Symptom Score (DVSS) questionnaire. Participants with $\geq 3$ missing items in the DVSS were excluded from all analyses. Males with greater than 9 points and females with greater than 6 points were defined as having $\operatorname{BBD}(17,18)$.

\section{Study design}

This was a single-centre cohort study (January 2016 to December 2019). The clinical data of 256 children with grade I-V VUR receiving CAP were analysed. Children who had experienced BT-UTI was eligible. Exposure were sex, younger age at the initial diagnosis of UTI $\leq 12$ months, high-grade VUR, bilateral VUR, aetiology, presence of renal scarring at the initial diagnosis, presence of renal function impairment at the initial diagnosis, ultrasound abnormalities, antibiotic used and BBD. Outcome was BT-UTI. The diagnostic criteria of BT-UTI included: (I) body temperature higher than $38^{\circ} \mathrm{C}$; cloudy, foul-smelling urine or symptoms such as urinary urgency, dysuria, and back pain; (II) abnormal midstream clean-catch urine laboratory values: leukocytes >5/high-power field and positive urinary leukocyte esterase; (III) urine culture: bacterial growth in midstream urine $>10^{5}$ colony-forming units $(\mathrm{CFU}) / \mathrm{mL}(18,19)$.

\section{Statistical analysis}

The primary endpoint was BT-UTI, which was analysed using a time to event model. The Kaplan-Meier method was used to compute the survival function and to build survival curves for a time to event analysis. Single-variable time-to-event analysis was performed for each exposure variable to determine the hazard ratio (HR) for the outcome (time to BT-UTI). Univariate analysis of significance for each prognostic factor is done by using log-rank test. A multivariable time-to-event model was then built by using a forward stepwise procedure. Variables with univariable $\mathrm{P}$ values $<0.05$ and high-grade VUR were considered for entry into the model. Cox regression was used to evaluate individual variables as independent risk factors for BT-UTI. Measurement data were expressed as the mean \pm standard deviation, and count data were presented as percentages (\%). Data analysis was performed with SPSS 22.0 software.

\section{IRB approval}

The study was conducted in accordance with the Declaration of Helsinki (as revised in 2013). The study was approved by Ethical Committee of Children Hospital of Fudan University (No. 2017-133) and written informed consent was provided by the participants' legal guardian/ next of kin.

\section{Results}

\section{General information}

A total of 256 children were included in this study, including 137 males and 119 females, with a median age of 8 (range, 1-56) months. The median follow-up time was 14.2 (range, 0-41) months. None of the male patients had been circumcised. All VUR children received MCU after being diagnosed with febrile UTI. Of these patients, 110 patients had unilateral VUR, 146 patients had bilateral VUR, and VUR was present in a total of 366 ureters. There were 13 children with grade I VUR (5.08\%), 42 grade II (16.41\%), 92 grade III (35.94\%), 86 grade IV (33.59\%), and 23 grade $\mathrm{V}(8.98 \%)$ (Table 1$)$.

\section{Risk factors for BT-UTI}

Among the 256 children, 81 developed BT-UTI while received CAP, for an incidence rate of $31.64 \%$. The median time of BT-UTI was 7 [3, 15] months. Univariate analysis found that younger age at the initial diagnosis of UTI ( $\leq 12$ months) $(\mathrm{P}=0.001)$, bilateral VUR $(\mathrm{P}=0.002)$, the presence of renal scarring at the initial diagnosis $(\mathrm{P}=0.001)$, and $\mathrm{BBD}(\mathrm{P}=0.014)$ were correlated with the occurrence of 
Table 1 Baseline demographic and clinical characteristics

\begin{tabular}{|c|c|c|c|}
\hline Characteristics & BT-UTI (n=81), n (\%) & Without BT-UTI (n=175), n (\%) & $\mathrm{P}$ \\
\hline Median, months & 4 & 6 & 0.212 \\
\hline Age group & & & 0.001 \\
\hline$\leq 12$ months & $62(24.21)$ & 97 (37.89) & \\
\hline Sex & & & 0.656 \\
\hline Male & $45(17.58)$ & 92 (35.94) & \\
\hline Female & $36(14.06)$ & $83(32.42)$ & \\
\hline \multicolumn{4}{|l|}{ VUR grade } \\
\hline$|-| I \mid$ & $42(16.41)$ & $105(41.02)$ & 0.220 \\
\hline IV-V & $39(15.23)$ & $70(27.34)$ & \\
\hline VUR & & & 0.002 \\
\hline Bilateral & $46(17.97)$ & $63(24.61)$ & \\
\hline Unilateral & $35(13.67)$ & $112(43.75)$ & \\
\hline Aetiology & & & 0.890 \\
\hline E. coli & $22(16.94)$ & $31(25.00)$ & \\
\hline Others & 29 (23.39) & $43(34.68)$ & \\
\hline Differential renal function $<45 \%$ & $28(10.55)$ & $70(27.34)$ & \\
\hline Ultrasound abnormalities & & & 0.491 \\
\hline Hydronephrosisg & $22(8.71)$ & $59(23.32)$ & \\
\hline Ureter duplication & $56(22.13)$ & $116(45.84)$ & \\
\hline BBD & & & 0.012 \\
\hline Yes & $13(24.07)$ & $8(14.81)$ & \\
\hline No & $9(16.67)$ & $24(44.44)$ & \\
\hline Antibiotic used & & & 0.660 \\
\hline Amoxicillin or cephalosporin & $60(23.43)$ & $125(48.83)$ & \\
\hline $\begin{array}{l}\text { Nitrofurantoin enteric-coatedtablets or } \\
\text { sulfamethoxazole tablets }\end{array}$ & $21(8.21)$ & $50(19.53)$ & \\
\hline
\end{tabular}

BT-UTI, breakthrough urinary tract infection; VUR, vesicoureteral reflux; E. coli, Escherichia coli Castellani; DMSA, dimercaptosuccinic acid; BBD, bladder and bowel dysfunction. 


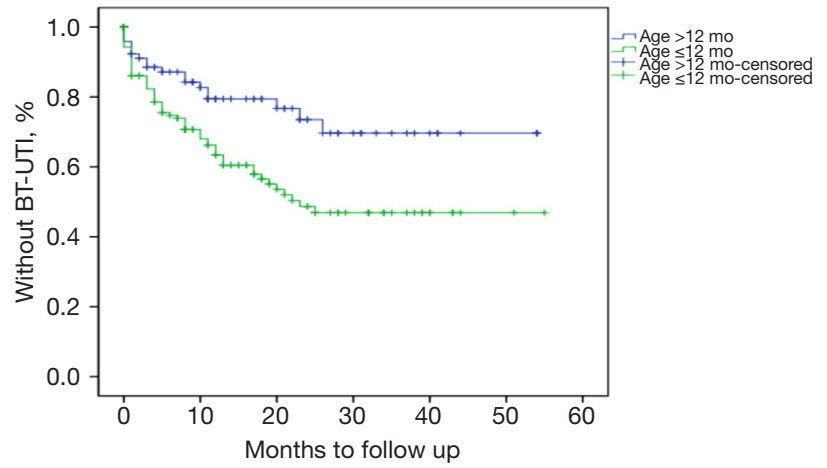

Figure 2 Time to first BT-UTI. Life-table analysis (Kaplan-Meier curves) showing the time of BT-UTI in VUR children with age at the initial diagnosis of UTI ( $\leq 12$ and $>12$ months). The difference was significant $(\mathrm{P}=0.001$, log-rank test). BT-UTI, breakthrough urinary tract infection; VUR, vesicoureteral reflux.

BT-UTI. The differences in other parameters such as the factor of sex between the BT-UTI and without BT-UTI groups were not significant. Multivariate analysis included the correlated factors with $\mathrm{P}<0.05$ in the univariate analysis and VUR grade. The results suggested that younger age at the initial diagnosis of UTI ( $\leq 12$ months) [HR: 4.629; 95\% confidence interval (CI): 1.302-16.462; $\mathrm{P}=0.001]$, bilateral VUR (HR: 2.078; 95\% CI: 1.084-4.022; $\mathrm{P}=0.002$ ) and BBD (HR: 3.194; 95\% CI: 1.243-8.206; $\mathrm{P}=0.014$ ) were independent risk factors for BT-UTI. Figure 2 shows the survival curve for the event BT-UTI for the two age groups. When children were divided into the high-grade group (III-V grade) and the low-grade group (I-II grade). High-grade VUR did not show any significant effect on the occurrence of BT-UTI on univariate analysis $(\mathrm{P}=0.152)$ in our group. Multivariate regression was performed, and no significant difference was observed neither (HR: 1.062; 95\% CI: 0.197-5.714; P=0.945). Moreover, when children were divided into grade IV-V and grade I-III groups, univariate analysis showed that grade IV-V VUR did not show significant effect on BT-UTI occurrence $(\mathrm{P}=0.221)$. Multivariate regression again showed no significant difference (HR: 1.194; 95\% CI: 0.460-3.099; $\mathrm{P}=0.716$ ) (Table 2).

A total of 54 VUR children who received potty training during the follow-up were evaluated with the DVSS questionnaire, including 33 males and 22 females. There were $21(38.89 \%)$ children with BBD, including 10 males and 11 females. Among these 21 patients with BBD, 2 were grade I-II VUR and the other 19 were grade III-V VUR.
Meanwhile, 12 were bilateral VUR. Chi-square test found that bilateral VUR was correlated with $\mathrm{BBD}(\mathrm{P}<0.05)$, while there was no difference in other parameters (sex, age and VUR grade) between the BBD and without BBD groups $(\mathrm{P}>0.05)$ (Table 3).

\section{Discussion}

In this study, the clinical data of 256 children with grade IV VUR receiving CAP at the Department of Nephrology, Children's Hospital of Fudan University were analysed. BT-UTI while receiving CAP was analyzed as outcome in a time-to-event model. Children who were younger at the initial diagnosis of UTI ( $\leq 12$ months), who had bilateral VUR and who had BBD were more prone to BT-UTI.

Despite receiving CAP and regular follow-up, some children with VUR still experienced BT-UTI. The incidence of BT-UTI was $31.64 \%$, and the incidence of more than one bout of BT-UTI was $14.11 \%$, which was consistent with the data of the cohort study (36.7\%) reported by Smellie et al. (20), which included 226 British VUR children receiving CAP. They are also similar to the incidence of symptomatic recurrent UTI reported in the International Reflux Study in Children, which performed 10 years of follow-up, during which the incidence of UTI (fever and non-fever) in the CAP group was $37.8 \%$ (21). Panaretto et al. (22) conducted a retrospective analysis of 290 children seeking treatment due to UTI in Australia and found that age less than 6 months and high-grade VUR were risk factors for BT-UTI. Dias et al. (23) performed a retrospective analysis of 740 children with grade I-V VUR who had received CAP and were followed up for at least 3 months in Brazil. They also found that younger age ( $\leq 12$ months) at the initial diagnosis of UTI, higher grade of VUR (III-V), BBD, and female sex were independent risk factors for BT-UTI. The incidence of BT-UTI in patients aged $\leq 12$ months was 2.4 times that of patients aged $>12$ months. In this study, the HR of BT-UTI in VUR children younger than 12 months $v s$. VUR children older than 12 months was 4.6, in line with the above two studies. In our study, there were only 4 boys who had BT-UTI beyond 12-month-old, while 15 girls with BT-UTI more than 1-year-old. The risk of BT-UTI decreases significantly after the first year of life in boys (24). In that way, discontinuation of antibiotic prophylaxis can be discussed, especially in boys with a low-grade reflux and normal renal parenchyma in DMSA scan. For girls, the risk for BT-UTI remains higher overall and the new scars were acquired and 
Table 2 Time-to-event analysis for risk of BT-UTI

\begin{tabular}{|c|c|c|c|c|c|c|}
\hline Variables & \multicolumn{3}{|c|}{ Univariable } & \multicolumn{3}{|c|}{ Multivariable } \\
\hline \multicolumn{7}{|l|}{ Age group } \\
\hline$\leq 12$ months & 2.624 & $1.449-4.753$ & 0.001 & 4.629 & $1.302-16.462$ & 0.018 \\
\hline$>12$ months & Ref. & & & & & \\
\hline$|-| \mid$ & Ref. & & & & & \\
\hline III-V & 1.652 & $0.831-3.284$ & 0.152 & 1.062 & $0.197-5.714$ & 0.945 \\
\hline I-III & Ref. & & & & & \\
\hline IV-V & 1.393 & $0.819-2.378$ & 0.221 & 1.194 & $0.460-3.099$ & 0.716 \\
\hline Unilateral & Ref. & & & & & \\
\hline \multicolumn{7}{|c|}{ DMSA at the initial diagnosis } \\
\hline Renal scarring & 2.843 & $1.563-5.172$ & 0.001 & 1.141 & $0.457-2.701$ & 0.817 \\
\hline Without renal scarring & Ref. & & & & & \\
\hline \multicolumn{7}{|l|}{ BBD } \\
\hline Yes & 4.333 & $1.349-3.924$ & 0.014 & 3.194 & $1.243-8.206$ & 0.016 \\
\hline No & Ref. & & & & & \\
\hline
\end{tabular}

BT-UTI, breakthrough urinary tract infection; HR, hazard ratio; CI, confidence interval; VUR, vesicoureteral reflux; DMSA, dimercaptosuccinic acid; BBD, bladder and bowel dysfunction.

found to be related to severe inflammatory processes, while in boys the renal damage was often congenital (24). Thus, prophylaxis also had a protective effect against new renal scarring in girls during 2 years of follow-up, but there were no effects on UTI recurrence or renal damage in boys (25). For girls the timing for CAP discontinuation should be extended after the patient is toilet-trained. Mattoo et al. (18) conducted a prospective randomized controlled clinical trial [Randomized Intervention for Vesicoureteral Reflux trial (RIVUR)] and found that children with grade IVV VUR were more prone to renal scarring and BT-UTI, which were 24.2 and 1.88 times the rates in grade I-III VUR children, respectively. In our study, neither univariate analysis nor multivariate analysis found a correlation between VUR grade and BT-UTI, but the results suggested that children with bilateral VUR were more likely to develop BT-UTI. This result may be related to the higher proportion of children with high-grade VUR included in this cohort $(78.52 \%$ of children with grade III-V VUR and $42.58 \%$ with grade IV-V VUR). Loukogeorgakis et al. (26) performed a retrospective analysis on 61 grade I-V VUR children, in which grade III-V VUR children were treated with CAP and grade I-II VUR children did not receive any treatment. The presence of renal scarring after the initial diagnosis of UTI was the only independent risk factor for the occurrence of BT-UTI (HR: 3.3; 95\% CI: 1.4-7.4). Signorelli et al. (1) analysed 732 children in two cohort studies, RIVUR and the Careful Urinary Tract Infection Evaluation (CUTIE) trial, and found that after the initial diagnosis of UTI, the recurrence rate of BTUTI in patients with renal scarring on DMSA scans was 2.88 times that of children without renal scarring. In this study by univariate analysis, the presence of renal scarring at the initial diagnosis is statistically significant, but in the multivariate analysis, it is not an independent risk factor for BT-UTI (HR: 1.141; 95\% CI: 0.457-2.701; $\mathrm{P}=0.817$ ).

BBD includes bladder and bowel dysfunction. In a cohort study, Burger et al. found that BBD was an independent risk 


\begin{tabular}{|c|c|c|c|}
\hline Characteristic & BBD $(n=21), n(\%)$ & No BBD $(n=33), n(\%)$ & $P$ \\
\hline Sex & & & 0.135 \\
\hline Male & $10(18.52)$ & $22(40.74)$ & \\
\hline Female & $11(20.37)$ & $11(20.37)$ & \\
\hline Age group & & & 0.381 \\
\hline$\leq 12$ months & $13(24.07)$ & 23 (42.59) & \\
\hline$>12$ months & $8(14.81)$ & $10(18.52)$ & \\
\hline \multicolumn{4}{|l|}{ VUR grade } \\
\hline I-II & $2(3.70)$ & $5(9.26)$ & \multirow{2}{*}{0.437} \\
\hline III-V & 19 (35.19) & 28 (51.85) & \\
\hline I-III & $10(18.52)$ & $18(33.33)$ & \multirow{2}{*}{0.414} \\
\hline IV-V & $11(20.37)$ & $15(27.78)$ & \\
\hline VUR & & & 0.028 \\
\hline Bilateral & $12(22.22)$ & $9(16.67)$ & \\
\hline Unilateral & $9(16.67)$ & $24(44.44)$ & \\
\hline
\end{tabular}

BBD, bladder and bowel dysfunction; VUR, vesicoureteral reflux.

factor for BT-UTI in VUR children receiving CAP (27). It was an independent risk factor for BT-UTI in children with VUR, and the risk of BT-UTI in VUR children with BBD while receiving CAP was 2.07 times that of VUR children without BBD. Shaikh et al. (28) analysed 802 children in the RIVUR and CUTIE cohort studies and found that VUR children with BBD were more prone to BT-UTI than VUR children without BBD. A second BT-UTI occurred in $51 \%$ of VUR children with BBD, while a second BT-UTI occurred in only $20 \%$ of VUR children without BBD. This study also found that although they received CAP, the HR of experiencing BT-UTI was 3.194 for VUR children with BBD vs. VUR children without BBD. Currently, BBD can be treated through potty training, animated biofeedback, sacral nerve stimulation, and active bowel management. Bilateral ureteral reimplantation can be performed for VUR children with BBD while CAP is provided, thereby reducing the rate of BT-UTI in clinical practice.

This study has some limitations. First, the study was a single-centre, small cohort study. Second, although the children were regularly followed up in our hospital, there was no quantitative standard for CAP compliance. In addition, there were some data defects during data acquisition, such as the lack of aetiology during the initial diagnosis of UTI. These factors may have led to biased results. Multicentre prospective clinical studies are needed to confirm the reliability of our results.

\section{Conclusions}

This study found that younger age at the initial diagnosis of UTI ( $\leq 12$ months), bilateral VUR and BBD were risk factors for BT-UTI recurrence. All of these factors need attention in clinical diagnosis and treatment.

\section{Acknowledgments}

Funding: This study was supported by Scientific Research Project of Shanghai Municipal Health and Family Planning Commission (No. 201740110) and Xiamen Health Guidance Project (No. 3502Z20209215).

\section{Footnote}

Reporting Checklist: The authors have completed the STROBE reporting checklist. Available at https:// tp.amegroups.com/article/view/10.21037/tp-21-398/rc

Data Sharing Statement: Available at https://tp.amegroups. com/article/view/10.21037/tp-21-398/dss

Peer Review File: Available at https://tp.amegroups.com/ article/view/10.21037/tp-21-398/prf

Conflicts of Interest: All authors have completed the ICMJE uniform disclosure form (available at https://tp.amegroups. com/article/view/10.21037/tp-21-398/coif). DS reports that this study was supported by Xiamen Health Guidance Project (No. 3502Z20209215). QS reports that this study was supported by Scientific Research Project of Shanghai Municipal Health and Family Planning Commission (No. 201740110). The other authors have no conflicts of interest to declare.

Ethical Statement: The authors are accountable for all aspects of the work in ensuring that questions related to the accuracy or integrity of any part of the work are appropriately investigated and resolved. The study was conducted in accordance with the Declaration of Helsinki (as revised in 2013). The study was approved by Ethical Committee of Children Hospital of Fudan University (No. 2017-133) and written informed consent was provided by 
the participants' legal guardian/next of kin.

Open Access Statement: This is an Open Access article distributed in accordance with the Creative Commons Attribution-NonCommercial-NoDerivs 4.0 International License (CC BY-NC-ND 4.0), which permits the noncommercial replication and distribution of the article with the strict proviso that no changes or edits are made and the original work is properly cited (including links to both the formal publication through the relevant DOI and the license). See: https://creativecommons.org/licenses/by-nc-nd/4.0/.

\section{References}

1. Signorelli $M$, Cerri V, Taddei F, et al. Prenatal diagnosis and management of mild fetal pyelectasis: implications for neonatal outcome and follow-up. Eur J Obstet Gynecol Reprod Biol 2005;118:154-9.

2. $\mathrm{Xu} \mathrm{J}, \mathrm{Xu} \mathrm{H}$, Zhou LJ, et al. Analysis of the prognosis and clinical factors in primary vesicoureteral reflux patients. Chinese Journal of Pediatrics 2012;50:587-92.

3. Simoes e Silva AC, Silva JM, Diniz JS, et al. Risk of hypertension in primary vesicoureteral reflux. Pediatr Nephrol 2007;22:459-62.

4. Silva JM, Diniz JS, Silva AC, et al. Predictive factors of chronic kidney disease in severe vesicoureteral reflux. Pediatr Nephrol 2006;21:1285-92.

5. DeMuri GP, Wald ER. Imaging and antimicrobial prophylaxis following the diagnosis of urinary tract infection in children. Pediatr Infect Dis J 2008;27:553-4.

6. Herz D, Merguerian P, McQuiston L. Continuous antibiotic prophylaxis reduces the risk of febrile UTI in children with asymptomatic antenatal hydronephrosis with either ureteral dilation, high-grade vesicoureteral reflux, or ureterovesical junction obstruction. J Pediatr Urol 2014;10:650-4.

7. Estrada CR Jr, Passerotti CC, Graham DA, et al. Nomograms for predicting annual resolution rate of primary vesicoureteral reflux: results from 2,462 children. J Urol 2009;182:1535-41.

8. Wang Z, Xu H, Liu HM, et al. Clinical analysis of 139 cases of primary vesicoureteric reflux in children. Chinese Journal of Pediatrics 2008;46:518-21.

9. Arlen AM, Kirsch AJ, Leong T, et al. Validation of the ureteral diameter ratio for predicting early spontaneous resolution of primary vesicoureteral reflux. J Pediatr Urol 2017;13:383.e1-6.

10. Anraku T, Obara K, Tasaki M, et al. Retrospective Analysis to Determine the Optimal Timing to Discontinue Continuous Antibiotic Prophylaxis in Patients with Primary Vesicoureteral Reflux. Urol Int 2019;102:462-7.

11. Mathews R, Mattoo TK. The role of antimicrobial prophylaxis in the management of children with vesicoureteral reflux--the RIVUR study outcomes. Adv Chronic Kidney Dis 2015;22:325-30.

12. Garin EH, Olavarria F, Garcia Nieto V, et al. Clinical significance of primary vesicoureteral reflux and urinary antibiotic prophylaxis after acute pyelonephritis: a multicenter, randomized, controlled study. Pediatrics 2006;117:626-32.

13. Wang HH, Gbadegesin RA, Foreman JW, et al. Efficacy of antibiotic prophylaxis in children with vesicoureteral reflux: systematic review and meta-analysis. J Urol 2015;193:963-9.

14. Medical versus surgical treatment of primary vesicoureteral reflux: report of the International Reflux Study Committee. Pediatrics 1981;67:392-400.

15. Piepsz A, Colarinha P, Gordon I, et al. Guidelines for 99mTc-DMSA scintigraphy in children. Eur J Nucl Med 2001;28:BP37-41.

16. Zhang $\mathrm{X}, \mathrm{Xu} \mathrm{H}$, Zhou L, et al. Accuracy of early DMSA scan for VUR in young children with febrile UTI. Pediatrics 2014;133:e30-8.

17. Farhat W, Bägli DJ, Capolicchio G, et al. The dysfunctional voiding scoring system: quantitative standardization of dysfunctional voiding symptoms in children. J Urol 2000;164:1011-5.

18. Mattoo TK, Chesney RW, Greenfield SP, et al. Renal Scarring in the Randomized Intervention for Children with Vesicoureteral Reflux (RIVUR) Trial. Clin J Am Soc Nephrol 2016;11:54-61.

19. Keren R, Shaikh N, Pohl H, et al. Risk Factors for Recurrent Urinary Tract Infection and Renal Scarring. Pediatrics 2015;136:e13-21.

20. Smellie JM, Prescod NP, Shaw PJ, et al. Childhood reflux and urinary infection: a follow-up of 10-41 years in 226 adults. Pediatr Nephrol 1998;12:727-36.

21. Jodal U, Smellie JM, Lax H, et al. Ten-year results of randomized treatment of children with severe vesicoureteral reflux. Final report of the International Reflux Study in Children. Pediatr Nephrol 2006;21:785-92.

22. Panaretto K, Craig J, Knight J, et al. Risk factors for recurrent urinary tract infection in preschool children. J Paediatr Child Health 1999;35:454-9.

23. Dias CS, Silva JM, Diniz JS, et al. Risk factors for recurrent urinary tract infections in a cohort of patients 
with primary vesicoureteral reflux. Pediatr Infect Dis J 2010;29:139-44.

24. Swerkersson S, Jodal U, Sixt R, et al. Relationship among vesicoureteral reflux, urinary tract infection and renal damage in children. J Urol 2007;178:647-51; discussion 650-1.

25. Brandström P, Esbjörner E, Herthelius M, et al. The Swedish reflux trial in children: III. Urinary tract infection pattern. J Urol 2010;184:286-91.

26. Loukogeorgakis SP, Burnand K, MacDonald A, et al. Renal

Cite this article as: Su D, Shen Q, Zhai Y, Chen J, Rao J, Miao Q, Tang X, Zhang Z, Liu J, Liu J, Xu H, Fang X. Risk factors for breakthrough urinary tract infection in children with vesicoureteral reflux receiving continuous antibiotic prophylaxis. Transl Pediatr 2022;11(1):1-9. doi: 10.21037/tp21-398 scarring is the most significant predictor of breakthrough febrile urinary tract infection in patients with simplex and duplex primary vesico-ureteral reflux. J Pediatr Urol 2020;16:189.e1-7.

27. Burgers R, de Jong TP, Visser M, et al. Functional defecation disorders in children with lower urinary tract symptoms. J Urol 2013;189:1886-91.

28. Shaikh N, Hoberman A, Keren R, et al. Recurrent Urinary Tract Infections in Children With Bladder and Bowel Dysfunction. Pediatrics 2016;137:e20152982. 